\title{
Perrin n-Dimensional Relations
}

\author{
Renata Passos Machado Vieira ${ }^{1 *}$, Milena Carolina dos Santos Mangueira ${ }^{1}$, Francisco Regis Vieira Alves ${ }^{1}$ and Paula \\ Maria Machado Cruz Catarino ${ }^{2}$ \\ ${ }^{1}$ Department of Mathematics, Federal Institute of Education, Science and Techonology of State of Ceara - IFCE \\ ${ }^{2}$ University of Trás-os-Montes and Alto Douro - UTAD \\ ${ }^{*}$ Corresponding author
}

\author{
Article Info \\ Keywords: n-dimensional relations, \\ Perrin sequence, Three-dimensional re- \\ lations, Two-dimensional relations \\ 2010 AMS: 11B36, $11 B 39$ \\ Received: 04 February 2021 \\ Accepted: 19 May 2021 \\ Available online: 05 June 2021
}

\begin{abstract}
This work aims, to perform a complexity in the Perrin sequence, to present the twodimensional, three-dimensional, and n-dimensional recurrence relations of this sequence. Thus, from the one-dimensional relationship of this sequence, we will discuss the increase of its dimensionality and the insertion of imaginary units in the Perrin sequence, which is a recursive sequence of third order and presents large similarities with the Padovan sequence, differing only its initial values. Moreover, we will present a relationship between the Perrin numbers and the Padovan numbers, which will be used to perform the complexity of this sequence.
\end{abstract}

\section{Introduction}

Perrin sequence was discovered by French engineer François Olivier Raoul Perrin (1841-1910), [1] affirms that this sequence was implicitly mentioned by mathematician Édouard Lucas in 1876, but only in 1899, François Perrin defined the Perrin sequence. This is linear sequence of third order is very similar to the Padovan sequence, since their recurrence formula are equal, changing only its initial values, so we will denote the Perrin numbers by $P e(n)$ and Padovan numbers by $\operatorname{Pd}(n)$.

The recurrence of the Perrin sequence is given by $P e(n)=P e(n-2)+P e(n-3), n \geq 3$, being $P e(0)=3, P e(1)=0$ and $P e(2)=2$ its initial values, presenting the first ones as: $3,0,2,3,2,5,5,7,10,12,17$. On the other hand, the Padovan initial values are $P d(0)=P d(1)=P d(2)=1$, maintaining the same recurrence than the Perrin sequence, we have that the first terms of this sequence is given by: $1,1,1,2,2,3,4,5,7,9,12$. Thus, from the similarity between these two sequences, [2] presents a relation between the Perrin numbers and the Padovan numbers given by:

$$
P e(n)=2 P d(n-4)+3 P d(n-5), n \geq 5 .
$$

It is noteworthy that this equation is derived from their respective matrix formulas of the Padovan and Perrin numbers [1] and [3]-[5] and will be used in the following sections.

In this sense, in this work, we will present the process of complexity of the Perrin sequence, which is associated with the insertion of the imaginary unit, the dimensional increase and its corresponding algebraic representation. The complexity process of this sequence is based on the work of [2] and [6]-[9]. Thus, the following sections will present the two-dimensional, three-dimensional, and n-dimensional relations of the Perrin sequence.

\section{Two-dimensional Perrin relations}

Initially, Harman [6] explores the two-dimensional relations or Gaussian numbers, denoted by $(n, m)=n+m i$, where $n$ and $m$ are integers and $i^{2}=-1$. Thus, from the one-dimensional Perrin recurrence and based on [2], in this section, we will increase 
the dimensionality of this sequence and insert the imaginary unit $i$, presenting then the two-dimensional relation of the Perrin sequence.

Definition 2.1. The numbers described in the form Pe $(n, m)$ will be represented by the numbers of the two-dimensional Perrin sequence, thus satisfying their respective two-dimensional recurrence conditions, where $n, m \in \mathbb{N}$ :

$$
\left\{\begin{array}{l}
P e(n+1, m)=P e(n-1, m)+P e(n-2, m) \\
P e(n, m+1)=P e(n, m-1)+P e(n, m-2)
\end{array}\right.
$$

Presenting the initial values defined as: $P e(0,0)=3, P e(1,0)=0, P e(0,1)=3+2 i, P e(0,2)=3+3 i, P e(2,0)=2, P e(2,1)=$ $2+2 i, P e(1,2)=3 i, P e(2,2)=2+3 i, P e(1,1)=2 i$ where $i^{2}=-1$ and $P e(0)=3, P e(1)=0, P e(2)=2$.

Lemma 2.2. Given the following properties:

(i) $P e(n, 0)=P e(n)$,

(ii) $\operatorname{Pe}(0, m)=3 P d(m)+P e(m+1) i$,

(iii) $P e(n, 1)=P e(n)+2 P d(n) i$,

(iv) $P e(1, m)=P e(m+1) i$.

Proof. According to $P e(n+1, m)=P e(n-1, m)+P e(n-2, m)$, having defined the initial values and applying the second principle of finite induction on $n$, where it fixes $m=0$ and varies $n=0,1,2, \ldots, k$, we observe:

$$
\begin{aligned}
P e(n+1,0) & =P e(n-1,0)+P e(n-2,0): \\
P e(3,0) & =P e(1,0)+P e(0,0)=3=P e(3) ; \\
P e(4,0) & =P e(2,0)+P e(1,0)=2=P e(4) ; \\
P e(5,0) & =P e(3,0)+P e(2,0)=5=P e(5) ; \\
\vdots & \\
P e(k-3,0) & =P e(k-5,0)+P e(k-6,0)=P e(k-3) ; \\
P e(k-2,0) & =P e(k-4,0)+P e(k-5,0)=P e(k-2) ; \\
P e(k-1,0) & =P e(k-3,0)+P e(k-4,0)=P e(k-1) ; \\
P e(k, 0) & =P e(k-2,0)+P e(k-3,0) \\
& =P e(k-2)+P e(k-3)=P e(k) .
\end{aligned}
$$

Thus, we can verify the property $P e(n, 0)=P e(n)$. Analogously, one can prove the validity $P e(0, m)=3 P d(m)+P e(m+1) i$, considering the relation $P e(n, m+1)=P e(n, m-1)+P e(n, m-2)$ also verifying the relation of the Perrin sequence with the Padovan sequence, and the initial Padovan numbers $P d(0)=P d(1)=P d(2)=1$. By analysing the recursiveness for $n=0$ and varying $m=0,1,2,3, \ldots, k$, we see that:

$$
\begin{aligned}
P e(0, m+1) & =P e(0, m-1)+P e(0, m-2): \\
P e(0,3) & =P e(0,1)+P e(0,0)=6+2 i=3 P d(3)+P e(4) i \\
P e(0,4) & =P e(0,2)+P e(0,1)=6+5 i=3 P d(4)+P e(5) i \\
P e(0,5) & =P e(0,3)+P e(0,2)=9+5 i=3 P d(5)+P e(6) i \\
\vdots & \\
P e(0, k-3) & =P e(0, k-5)+P e(0, k-6)=3 P d(k-3)+P e(k-2) i \\
P e(0, k-2) & =P e(0, k-4)+P e(0, k-5)=3 P d(k-2)+P e(k-1) i \\
P e(0, k-1) & =P e(0, k-3)+P e(0, k-4)=3 P d(k-1)+P e(k) i \\
P e(0, k) & =P e(0, k-2)+P e(0, k-3) \\
& =3 P d(k-2)+P e(k-1) i+3 P d(k-3)+P e(k-2) i=3 P d(k)+P e(k+1) i .
\end{aligned}
$$

Validating the property $P e(0, m)=3 P d(m)+P e(m+1) i$. To demonstrate the following property, the same principle of induction is used, with: $P e(n, m+1)=P e(n, m-1)+P e(n, m-2)$ and with the initial values established at the beginning. We also verify a relationship between the Perrin sequence and the Padovan sequence, thus fixing $m=1$ and varying $n=0,1,2,3, \ldots, k$, 
it follows that:

$$
\begin{aligned}
P e(n+1,1) & =P e(n-1,1)+P e(n-2,1): \\
P e(3,1) & =P e(1,1)+P e(0,1)=3+4 i=P e(3)+2 P d(3) i ; \\
P e(4,1) & =P e(2,1)+P e(1,1)=2+4 i=P e(4)+2 P d(4) i ; \\
P e(5,1) & =P e(3,1)+P e(2,1)=5+6 i=P e(5)+2 P d(5) i ; \\
\vdots & \\
P e(k-3,1) & =P e(k-5,1)+P e(k-6,1)=P e(k-3)+2 P d(k-3) i ; \\
P e(k-2,1) & =P e(k-4,1)+P e(k-5,1)=P e(k-2)+2 P d(k-2) i ; \\
P e(k-1,1) & =P e(k-3,1)+P e(k-4,1)=P e(k-1)+2 P d(k-1) i ; \\
P e(k, 1) & =P e(k-2,1)+P e(k-3,1) \\
& =P e(k-2)+2 P d(k-2) i+P e(k-3)+2 P d(k-3) i=P e(k)+2 P d(k) i .
\end{aligned}
$$

Proving that $P e(n, 1)=P e(n)+2 P d(n) i$. Concluding the properties demonstrations, we have that, analogously, we can consider the relation $P e(n, m+1)=P e(n, m-1)+P e(n, m-2)$, the values established initially, and fixing $n=1$ and varying $m=0,1,2,3, \ldots, k$. We note that:

$$
\begin{aligned}
P e(1, m+1) & =P e(1, m-1)+P e(1, m-2): \\
P e(1,3) & =P e(1,1)+P e(1,0)=2 i=P e(4) i ; \\
P e(1,4) & =P e(1,2)+P e(1,1)=5 i=P e(5) i ; \\
P e(1,5) & =P e(1,3)+P e(1,2)=5 i=P e(6) i ; \\
\vdots & \\
P e(1, k-3) & =P e(1, k-5)+P e(1, k-6)=P e(k-2) i ; \\
P e(1, k-2) & =P e(1, k-4)+P e(1, k-5)=P e(k-1) i ; \\
P e(1, k-1) & =P e(1, k-3)+P e(1, k-6)=P e(k) i ; \\
P e(1, k) & =P e(1, k-2)+P e(1, k-3) \\
& =P e(k-1) i+P e(k-2) i=P e(k+1) i .
\end{aligned}
$$

Theorem 2.3. For the two integers, $n, m \in \mathbb{N}$, the numbers in the form $P e(n, m)$ are described by:

$$
P e(n, m)=P e(n) P d(m)+P e(m+1) P d(n) i .
$$

Proof. Fixing the value of natural number $n$, we can carry out the demonstration by induction on $m$. For $m=0$, there is the property $P e(n, 0)=P e(n)$ and $P e(n, 1)=P e(n)+2 P d(n) i$, previously validated by Lemma 2.2 , where $P e(0)=3, P e(1)=$ $0, P e(2)=2$, whose initial values were defined previously. For this, some values of $P e(n, m)$ will be calculated, varying $m$. For $P e(n, 2)$, we use the recurrence $P e(n, m+1)=P e(n, m-1)+P e(n, m-2)$ with the initial values established, with $m=2$ fixed and $n=0,1,2,3, \ldots, k$, we have that:

$$
\begin{aligned}
P e(n+1,2) & =P e(n-1,2)+P e(n-2,2): \\
P e(3,2) & =P e(1,2)+P e(0,2)=3+6 i=P e(3)+3 P d(3) i \\
P e(4,2) & =P e(2,2)+P e(1,2)=2+6 i=P e(4)+3 P d(4) i ; \\
P e(5,2) & =P e(3,2)+P e(2,2)=5+9 i=P e(5)+3 P d(5) i \\
\vdots & \\
P e(k-3,2) & =P e(k-5,2)+P e(k-6,2)=P e(k-3)+3 P d(k-3) i \\
P e(k-2,2) & =P e(k-4,2)+P e(k-5,2)=P e(k-2)+3 P d(k-2) i ; \\
P e(k-1,2) & =P e(k-3,2)+P e(k-4,2)=P e(k-1)+3 P d(k-1) i ; \\
P e(k, 2) & =P e(k-2,2)+P e(k-3,2) \\
& =P e(k-2)+3 P d(k-2) i+P e(k-3)+3 P d(k-3) i=P e(k)+3 P d(k) i .
\end{aligned}
$$

Rewriting the properties seen in the previous Lemma (i) and (ii), we have that:

$$
\begin{aligned}
& P e(n, 0)=P e(n)+\operatorname{Pe}(1) P d(n) i ; \\
& P e(n, 1)=\operatorname{Pe}(n)+\operatorname{Pe}(2) P d(n) i ;
\end{aligned}
$$


However, assuming that for $m=1,2, \ldots, k$, the following identities are valid:

$$
\begin{gathered}
P e(n, 0)=P e(n) P d(0)+P e(1) P d(n) i ; \\
P e(n, 1)=P e(n) P d(1)+P e(2) P d(n) i ; \\
P e(n, 2)=P e(n) P d(2)+P e(3) P d(n) i ; \\
\vdots \\
P e(n, k-3)=P e(n) P d(k-3)+P e(k-2) P d(n) i ; \\
P e(n, k-2)=P e(n) P d(k-2)+P e(k-1) P d(n) i ; \\
P e(n, k-1)=P e(n) P d(k-1)+P e(k) P d(n) i ; \\
P e(n, k)=P e(n, k-2)+P e(n, k-3) \\
=P e(n) P d(k-2)+P e(k-1) P d(n) i+P e(n) P d(k-3)+P e(k-2) P d(n) i \\
=P e(n) P d(k)+P e(k+1) P d(n) i
\end{gathered}
$$

Demonstrating for $m=k+1$, from the recurrence $P e(n, k+1)=P e(n, k-1)+P e(n, k-2)$, we have that:

$$
\begin{aligned}
\operatorname{Pe}(n, k+1) & =\operatorname{Pe}(n, k-1)+\operatorname{Pe}(n, k-2) \\
& =\operatorname{Pe}(n) \operatorname{Pd}(k-1)+\operatorname{Pe}(k) \operatorname{Pd}(n) i+\operatorname{Pe}(n) \operatorname{Pd}(k-2)+\operatorname{Pe}(k-1) \operatorname{Pd}(n) i \\
& =\operatorname{Pe}(n) \operatorname{Pd}(k+1)+\operatorname{Pe}(k+2) \operatorname{Pd}(n) i
\end{aligned}
$$

\section{The three-dimensional Perrin relations}

In this section, the three-dimensional Perrin relations will be presented, denoted by $P e(n, m, p)$, from its one-dimensional and two-dimensional recurrence. For this, we will increase the dimensionality of this sequence and insert the imaginary unit $i$ and $j$, where $i^{2}=j^{2}=-1$.

Definition 3.1. The Perrin numbers, we can consider the initial values, defined as: $P e(0,0,0)=3=P e(0), P e(1,0,0)=0=$ $P e(1), P e(2,0,0)=2=P e(2), P e(0,1,0)=3+2 i, P e(0,2,0)=3+3 i, P e(0,0,1)=3+2 j, P e(0,0,2)=3+3 j, P e(1,0,1)=$ $2 j, P e(1,0,2)=3 j, P e(1,1,0)=2 i, P e(1,2,0)=3 i, P e(0,1,1)=3+2 i+2 j, P e(0,1,2)=3+2 i+3 j, P e(0,2,1)=3+3 i+$ $2 j, P e(0,2,2)=3+3 i+3 j, P e(2,1,1)=2+2 i+2 j, P e(2,2,1)=2+3 i+2 j, P e(2,0,1)=2+2 j, P e(2,0,2)=2+3 j, P e(2,1,0)=$ $2+2 i, P e(2,2,0)=2+3 i, P e(1,1,1)=2 i+2 j, P e(1,2,1)=3 i+2 j, P e(1,1,2)=2 i+3 j$ in which $i^{2}=j^{2}=-1$, forming the numbers as $\mathrm{Pe}(n, m, p)$ satisfying the following three-dimensional recurrence conditions, where $n, m, p \geqslant 0$ :

$$
\left\{\begin{array}{l}
P e(n, m, p)=P e(n-2, m, p)+P e(n-3, m, p) \\
P e(n, m, p)=P e(n, m-2, p)+P e(n, m-3, p) \\
P e(n, m, p)=P e(n, m, p-2)+P e(n, m, p-3)
\end{array}\right.
$$

Lemma 3.2. The following properties are valid for Perrin numbers:
(a) $\mathrm{Pe}(n, 0,0)=\mathrm{Pe}(n)$,
(b) $P e(n, 1,0)=P e(n)+2 P d(n) i$,
(c) $\operatorname{Pe}(n, 0,1)=\operatorname{Pe}(n)+2 P d(n) j$,
(d) $P e(n, 1,1)=P e(n)+2 P d(n) i+2 P d(n) j$.

Proof. To demonstrate property (a) $P e(n, 0,0)=P e(n)$, we will consider the relation $P e(n, m, p)=P e(n-2, m, p)+P e(n-$ $3, m, p)$ and the initial values first defined. Thus, for $m=p=0$ and varying $n=(0,1,2,3, \ldots, k)$. We can see that:

$$
\begin{aligned}
P e(n, 0,0) & =P e(n-2,0,0)+P e(n-3,0,0): \\
P e(3,0,0) & =P e(1,0,0)+P e(0,0,0)=3=P e(3) ; \\
P e(4,0,0) & =P e(2,0,0)+P e(1,0,0)=2=P e(4) ; \\
P e(5,0,0) & =P e(3,0,0)+P e(2,0,0)=5=P e(5) ; \\
\vdots & \\
P e(k-3,0,0) & =P e(k-5,0,0)+P e(k-6,0,0)=P e(k-3) ; \\
P e(k-2,0,0) & =P e(k-4,0,0)+P e(k-5,0,0)=P e(k-2) ; \\
P e(k-1,0,0) & =P e(k-3,0,0)+P e(k-4,0,0)=P e(k-1) ; \\
P e(k, 0,0) & =P e(k-2,0,0)+P e(k-3,0,0) \\
& =P e(k-2)+P e(k-3)=P e(k) .
\end{aligned}
$$


Now, to validate property (b) $P e(n, 1,0)=P e(n)+2 P d(n) i$, we use the relation $P e(n, m, p)=P e(n-2, m, p)+P e(n-3, m, p)$. Therefore, the recursiveness for $m=p=1$, where $n=(0,1,2,3, \ldots, k)$, is such that:

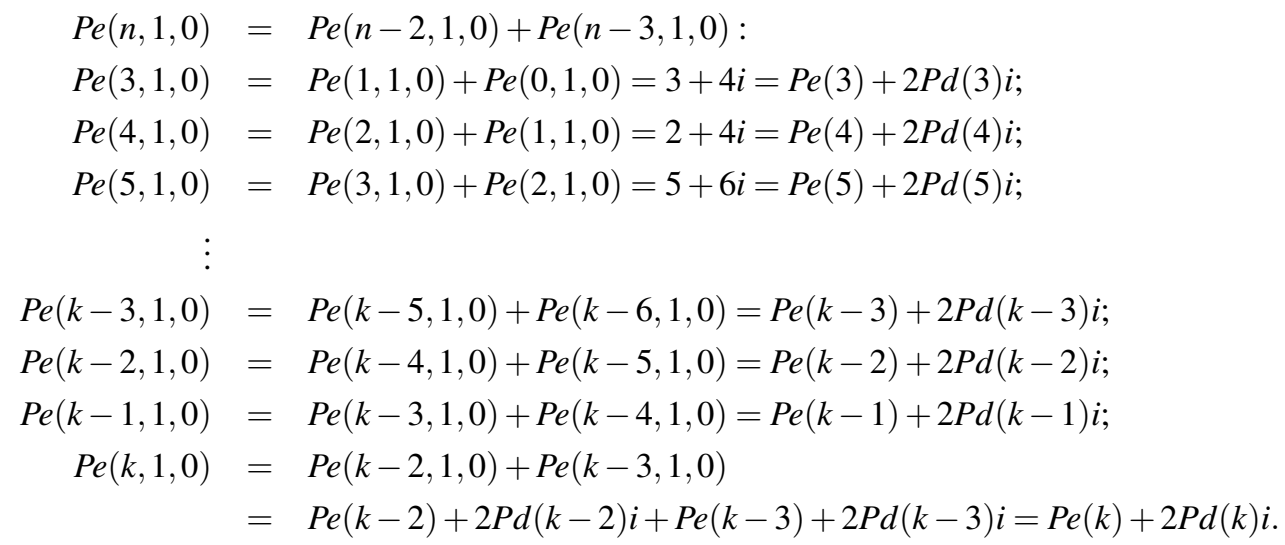

To demonstrate the third property (c) $P e(n, 0,1)=P e(n)+2 P d(n) j$ with $P e(n, m, p)=P e(n-2, m, p)+P e(n-3, m, p)$ we evaluate the recurrence for $m=0$ and $p=1$, where $n=(0,1,2,3, \ldots, k)$. Thus:

$$
\begin{aligned}
P e(n, 0,1) & =P e(n-2,0,1)+P e(n-3,0,1): \\
P e(3,0,1) & =P e(1,0,1)+P e(0,0,1)=3+4 j=P e(3)+2 P d(3) j ; \\
P e(4,0,1) & =P e(2,0,1)+P e(1,0,1)=2+4 j=P e(4)+2 P d(4) j ; \\
P e(5,0,1) & =P e(3,0,1)+P e(2,0,1)=5+6 j=P e(5)+2 P d(5) j ; \\
\vdots & \\
P e(k-3,0,1) & =P e(k-5,0,1)+P e(k-6,0,1)=P e(k-3)+2 P d(k-3) j ; \\
P e(k-2,0,1) & =P e(k-4,0,1)+P e(k-5,0,1)=P e(k-2)+2 P d(k-2) j ; \\
P e(k-1,0,1) & =P e(k-3,0,1)+P e(k-4,0,1)=P e(k-1)+2 P d(k-1) j ; \\
P e(k, 0,1) & =P e(k-2,0,1)+P e(k-3,0,1) \\
& =P e(k-2)+2 P d(k-2) j+P e(k-3)+2 P d(k-3) j \\
& =P e(k)+2 P d(k) j .
\end{aligned}
$$

Finally, by induction, we can demonstrate the property (d) $P e(n, 1,1)=P e(n)+2 P d(n) i+2 P d(n) j$ through the recurrence $P e(n, m, p)=P e(n-2, m, p)+P e(n-3, m, p)$ for $m=1$ and $p=1$, with variation $n=(0,1,2,3, \ldots, k)$. With this, we can see that:

$$
\begin{aligned}
P e(n, 1,1) & =P e(n-2,1,1)+P e(n-3,1,1): \\
P e(3,1,1) & =P e(1,1,1)+P e(0,1,1)=3+4 i+4 j=P e(3)+2 P d(3) i+2 P d(3) j ; \\
P e(4,1,1) & =P e(2,1,1)+P e(1,1,1)=2+4 i+4 j=P e(4)+2 P d(4) i+2 P d(4) j ; \\
P e(5,1,1) & =P e(3,1,1)+P e(2,1,1)=5+6 i+6 j=P e(5)+2 P d(5) i+2 P d(5) j ; \\
\vdots & \\
P e(k-3,1,1) & =P e(k-5,1,1)+P e(k-6,1,1)=P e(k-3)+2 P d(k-3) i+2 P d(k-3) j ; \\
P e(k-2,1,1) & =P e(k-4,1,1)+P e(k-5,1,1)=P e(k-2)+2 P d(k-2) i+2 P d(k-2) j ; \\
P e(k-1,1,1) & =P e(k-3,1,1)+P e(k-4,1,1)=P e(k-1)+2 P d(k-1) i+2 P d(k-1) j ; \\
P e(k, 1,1) & =P e(k-2,1,1)+P e(k-3,1,1) \\
& =P e(k-2)+2 P d(k-2) i+2 P d(k-2) j+P e(k-3)+2 P d(k-3) i+2 P d(k-3) j \\
& =P e(k)+2 P d(k) i+2 P d(k) j .
\end{aligned}
$$

Thus, the properties described above are verified.

Lemma 3.3. Given the following properties:

(a) $P e(0, m, 0)=3 P d(m)+P e(m+1) i$,

(b) $P e(0, m, 1)=3 P d(m)+P e(m+1) i+2 P d(m) j$,

(c) $P e(1, m, 0)=P e(m+1) i$,

(d) $P e(1, m, 1)=P e(m+1) i+2 P d(m) j$. 
Proof. (a) Given the recurrence, the second principle of induction on $m$ at $n=p=0$ applies. Thus, varying $m=1,2,3, \ldots, k$, we see that:

$$
\begin{aligned}
P e(0, m, 0) & =P e(0, m-2,0)+P e(0, m-3,0): \\
P e(0,3,0) & =P e(0,1,0)+P e(0,0,0)=6+2 i=3 P d(3)+P e(4) i ; \\
P e(0,4,0) & =P e(0,2,0)+P e(0,1,0)=6+5 i=3 P d(4)+P e(5) i ; \\
P e(0,5,0) & =P e(0,3,0)+P e(0,2,0)=9+5 i=3 P d(5)+P e(6) i ; \\
\vdots & \\
P e(0, k-3,0) & =P e(0, k-5,0)+P e(0, k-6,0)=3 P d(k-3)+P e(k-2) i ; \\
P e(0, k-2,0) & =P e(0, k-4,0)+P e(0, k-5,0)=3 P d(k-2)+P e(k-1) i ; \\
P e(0, k-1,0) & =P e(0, k-3,0)+P e(0, k-4,0)=3 P d(k-1)+P e(k) i ; \\
P e(0, k, 0) & =P e(0, k-2,0)+P e(0, k-3,0) \\
& =3 P d(k-2)+P e(k-1) i+3 P d(k-3)+P e(k-2) i \\
& =3 P d(k)+P e(k+1) i .
\end{aligned}
$$

Validating the property (a) $P e(0, m, 0)=3 P d(m)+P e(m+1) i$.

(b) For the demonstration of item (b), the same principle on $m$ at $n=0$ and $p=1$ follows. Hence, $m=k$ :

$$
\begin{aligned}
P e(0, m, 1) & =P e(0, m-2,1)+P e(0, m-3,1): \\
P e(0,3,1) & =P e(0,1,1)+P e(0,0,1)=6+2 i+4 j=3 P d(3)+P e(4) i+2 P d(3) j ; \\
P e(0,4,1) & =P e(0,2,1)+P e(0,1,1)=6+5 i+4 j=3 P d(4)+P e(5) i+2 P d(4) j ; \\
P e(0,5,1) & =P e(0,3,1)+P e(0,2,1)=9+5 i+6 j=3 P d(5)+P e(6) i+2 P d(5) j ; \\
\vdots & \\
P e(0, k-3,1) & =P e(0, k-5,1)+P e(0, k-6,1)=3 P d(k-3)+P e(k-2) i+2 P d(k-3) j ; \\
P e(0, k-2,1) & =P e(0, k-4,1)+P e(0, k-5,1)=3 P d(k-2)+P e(k-1) i+2 P d(k-2) j ; \\
P e(0, k-1,1) & =P e(0, k-3,1)+P e(0, k-4,1)=3 P d F(k-1)+P e(k) i+2 P d(k-1) j ; \\
P e(0, k, 1) & =P e(0, k-2,1)+P e(0, k-3,1) \\
& =3 P d(k-2)+P e(k-1) i+2 P d(k-2) j+3 P d(k-3)+P e(k-2) i+2 P d(k-3) j \\
& =3 P d(k)+P e(k+1) i+2 P d(k) j .
\end{aligned}
$$

Validating the property (b) $P e(0, m, 1)=3 P d(m)+P e(m+1) i+2 P d(m) j$.

(c) Following the same principle on $m$ at $n=1$ and $p=0$. Therefore, for $m=k$, we have that:

$$
\begin{aligned}
P e(1, m, 0) & =P e(1, m-2,0)+P e(1, m-3,0): \\
P e(1,3,0) & =P e(1,1,0)+P e(1,0,0)=2 i=P e(4) i ; \\
P e(1,4,0) & =P e(1,2,0)+P e(1,1,0)=5 i=P e(5) i ; \\
P e(1,5,0) & =P e(1,3,0)+P e(1,2,0)=5 i=P e(6) i ; \\
\vdots & \\
P e(1, k-3,0) & =P e(1, k-5,0)+P e(1, k-6,0)=P e(k-2) i ; \\
P e(1, k-2,0) & =P e(1, k-4,0)+P e(1, k-5,0)=P e(k-1) i ; \\
P e(1, k-1,0) & =P e(1, k-3,0)+P e(1, k-4,0)=P e(k) i ; \\
P e(1, k, 0) & =P e(1, k-2,0)+P e(1, k-3,0) \\
& =P e(k-1) i+P e(k-2) i=P e(k+1) i .
\end{aligned}
$$

Demonstrating the property (c) $P e(1, m, 0)=P e(m+1) i$. 
(d) Analogously, the same principle on $m$ at $n=p=1$ follows. Therefore, for $m=k$, we have that:

$$
\begin{aligned}
P e(1, m, 1) & =P e(1, m-2,1)+P e(1, m-3,1): \\
P e(1,3,1) & =P e(1,1,1)+P e(1,0,1)=2 i+4 j=P e(4) i+2 P d(3) j \\
P e(1,4,1) & =P e(1,2,1)+P e(1,1,1)=5 i+4 j=P e(5) i+2 P d(4) j \\
P e(1,5,1) & =P e(1,3,1)+P e(1,2,1)=5 i+6 j=P e(6) i+2 P d(5) j \\
\vdots & \\
P e(1, k-3,1) & =P e(1, k-5,1)+P e(1, k-6,1)=P e(k-2) i+2 P d(k-3) j \\
P e(1, k-2,1) & =P e(1, k-4,1)+P e(1, k-5,1)=P e(k-1) i+2 P d(k-2) j ; \\
P e(1, k-1,1) & =P e(1, k-3,1)+P e(1, k-4,1)=P e(k) i+2 P d(k-1) j \\
P e(1, k, 1) & =P e(1, k-2,1)+P e(1, k-3,1) \\
& =P e(k-1) i+2 P d(k-2) j+P e(k-2) i+2 P d(k-3) j \\
& =P e(k+1) i+2 P d(k) j .
\end{aligned}
$$

Therefore, property (d) $P e(1, m, 1)=P e(m+1) i+2 P d(m) j$.

Lemma 3.4. The following identities are valid:
(a) $\operatorname{Pe}(0,0, p)=3 P d(p)+P e(p+1) j$,
(b) $P e(0,1, p)=3 P d(p)+2 P d(p) i+P e(p+1) j$,
(c) $P e(1,0, p)=P e(p+1) j$,
(d) $P e(1,1, p)=2 P d(p) i+P e(p+1) j$.

Proof. (a) By applying the second principle of induction on $p$ for $n=m=0$ and varying $p=1,2,3, \ldots, k$, we have that:

$$
\begin{aligned}
P e(0,0, p) & =P e(0,0, p-2)+P e(0,0, p-3): \\
P e(0,0,3) & =P e(0,0,1)+P e(0,0,0)=6+2 j=3 P d(3)+P e(4) j ; \\
P e(0,0,4) & =P e(0,0,2)+P e(0,0,1)=6+5 j=3 P d(4)+P e(5) j ; \\
P e(0,0,5) & =P e(0,0,3)+P e(0,0,2)=9+5 j=3 P d(5)+P e(6) j ; \\
\vdots & \\
P e(0,0, k-3) & =P e(0,0, k-5)+P e(0,0, k-6)=3 P d(k-3)+P e(k-2) j ; \\
P e(0,0, k-2) & =P e(0,0, k-4)+P e(0,0, k-5)=3 P d(k-2)+P e(k-1) j ; \\
P e(0,0, k-1) & =P e(0,0, k-3)+P e(0,0, k-4)=3 P d(k-1)+P e(k) j ; \\
P e(0,0, k) & =P e(0,0, k-2)+P e(0,0, k-3) \\
& =3 P d(k-2)+P e(k-1) j+3 P d(k-3)+P e(k-2) j \\
& =3 P d(k)+P e(k+1) j .
\end{aligned}
$$

The property (a) $P e(0,0, p)=3 P d(p)+P e(p+1) j$ is, thus, validated.

(b) By using the second principle of induction on $p$ for $n=0$ and $m=1$ and varying $p=1,2,3, \ldots, k$, we have that:

$$
\begin{aligned}
P e(0,1, p) & =P e(0,1, p-2)+P e(0,1, p-3): \\
P e(0,1,3) & =P e(0,1,1)+P e(0,1,0)=6+4 i+2 j=3 P d(3)+2 P d(3) i+P e(4) j ; \\
P e(0,1,4) & =P e(0,1,2)+P e(0,1,1)=6+4 i+5 j=3 P d(4)+2 P d(4) i+P e(5) j ; \\
P e(0,1,5) & =P e(0,1,3)+P e(0,1,2)=9+6 i+5 j=3 P d(5)+2 P d(5) i+P e(6) j ; \\
\vdots & \\
P e(0,1, k-3) & =P e(0,1, k-5)+P e(0,1, k-6)=3 P d(k-3)+2 P d(k-3) i+P e(k-2) j ; \\
P e(0,1, k-2) & =P e(0,1, k-4)+P e(0,1, k-5)=3 P d(k-2)+2 P d(k-2) i+P e(k-1) j ; \\
P e(0,1, k-1) & =P e(0,1, k-3)+P e(0,1, k-4)=3 P d(k-1)+2 P d(k-1) i+P e(k) j ; \\
P e(0,1, k) & =P e(0,1, k-2)+P e(0,1, k-3) \\
& =3 P d(k-2)+2 P d(k-2) i+P e(k-1) j+3 P d(k-3)+2 P d(k-3) i+P e(k-2) j \\
& =3 P d(k)+2 P d(k) i+P e(k+1) j .
\end{aligned}
$$

Thus, the property (b) $P e(0,1, p)=3 P d(p)+2 P d(p) i+P e(p+1) j$ is validated. 
(c)Through the second principle of induction on $p$ for $n=1$ and $m=0$ and varying $p=1,2,3, \ldots, k$, we have that:

$$
\begin{aligned}
P e(1,0, p) & =P e(1,0, p-2)+P e(1,0, p-3): \\
P e(1,0,3) & =P e(1,0,1)+P e(1,0,0)=2 j=P e(4) j ; \\
P e(1,0,4) & =P e(1,0,2)+P e(1,0,1)=5 j=P e(5) j ; \\
P e(1,0,5) & =P e(1,0,3)+P e(1,0,2)=5 j=P e(6) j ; \\
\vdots & \\
P e(1,0, k-3) & =P e(1,0, k-5)+P e(1,0, k-6)=P e(k-2) j ; \\
P e(1,0, k-2) & =P e(1,0, k-4)+P e(1,0, k-5)=P e(k-1) j ; \\
P e(1,0, k-1) & =P e(1,0, k-3)+P e(1,0, k-4)=P e(k) j ; \\
P e(1,0, k) & =P e(1,0, k-2)+P e(1,0, k-3) \\
& =P e(k-1) j+P e(k-2) j=P e(k+1) j .
\end{aligned}
$$

Validating property (c) $P e(1,0, p)=P e(p+1) j$.

(d) According to the second principle of induction on $p$ for $n=m=1$ and varying $p=1,2,3, \ldots, k$, we have that:

$$
\begin{aligned}
P e(1,1, p) & =P e(1,1, p-2)+P e(1,1, p-3): \\
P e(1,1,3) & =P e(1,1,1)+P e(1,1,0)=4 i+2 j=2 P d(3) i+P e(4) j \\
P e(1,1,4) & =P e(1,1,2)+P e(1,1,1)=4 i+5 j=2 P d(4) i+P e(5) j \\
P e(1,1,5) & =P e(1,1,3)+P e(1,1,2)=6 i+5 j=2 P d(5) i+P e(6) j \\
\vdots & \\
P e(1,1, k-3) & =P e(1,1, k-5)+P e(1,1, k-6)=2 P d(k-3) i+P e(k-2) j \\
P e(1,1, k-2) & =P e(1,1, k-4)+P e(1,1, k-5)=2 P d(k-2) i+P e(k-1) j \\
P e(1,1, k-1) & =P e(1,1, k-3)+P e(1,1, k-4)=2 P d(k-1) i+P e(k) j \\
P e(1,1, k) & =P e(1,1, k-2)+P e(1,1, k-3) \\
& =2 P d(k-2) i+P e(k-1) j+2 P d(k-3) i+P e(k-2) j \\
& =2 P d(k) i+P e(k+1) j .
\end{aligned}
$$

Demonstrating property $(\mathrm{d}) P e(1,1, p)=2 P d(p) i+P e(p+1) j$.

Theorem 3.5. For the three integers, $n, m, p \in \mathbb{N}$, the numbers in the form $P e(n, m, p)$ are described by:

$$
P e(n, m, p)=\operatorname{Pe}(n) P d(m) P d(p)+P d(n) P e(m+1) P d(p) i+P d(n) P d(m) P e(p+1) j .
$$

Proof. Hence, for $p=0$ and $m=2$, we have that:

$$
\begin{aligned}
P e(3,2,0) & =P e(1,2,0)+P e(0,2,0)=3+6 i=P e(3)+3 P d(3) i ; \\
P e(4,2,0) & =P e(2,2,0)+P e(1,2,0)=2+6 i=P e(4)+3 P d(4) i ; \\
P e(5,2,0) & =P e(3,2,0)+P e(2,2,0)=5+9 i=P e(5)+3 P d(5) i ; \\
\vdots & \\
P e(n-3,2,0) & =P e(n-5,2,0)+P e(n-6,2,0)=P e(n-3)+3 P d(n-3) i ; \\
P e(n-2,2,0) & =P e(n-4,2,0)+P e(n-5,2,0)=P e(n-2)+3 P d(n-2) i ; \\
P e(n-1,2,0) & =P e(n-3,2,0)+P e(n-4,2,0)=P e(n-1)+3 P d(n-1) i ; \\
P e(n, 2,0) & =P e(n-2,2,0)+P e(n-3,2,0) \\
& =P e(n-2)+3 P d(n-2) i+P e(n-3)+3 P d(n-3) i \\
& =P e(n)+3 P d(n) i .
\end{aligned}
$$


In addition, are stimulated other properties inherent to this process, for $m=1,2,3, \ldots, k$, and we obtain:

$$
\begin{aligned}
P e(n, 0,0) & =P e(n)+P e(1) P d(n) i \\
P e(n, 1,0) & =P e(n)+P e(2) P d(n) i \\
P e(n, 2,0) & =P e(n)+P e(3) P d(n) i \\
P e(n, 3,0) & =P e(n, 1,0)+P e(n, 0,0)=2 P e(n)+P e(4) P d(n) i \\
& =P d(3) P e(n)+P e(4) P d(n) i ; \\
\vdots & \\
P e(n, k-3,0) & =P e(n, k-5,0)+P e(n, k-6,0)=P d(k-3) P e(n)+P e(k-2) P d(n) i ; \\
P e(n, k-2,0) & =P e(n, k-4,0)+P e(n, k-5,0)=P d(k-2) P e(n)+P e(k-1) P d(n) i ; \\
P e(n, k-1,0) & =P e(n, k-3,0)+P e(n, k-4,0)=P d(k-1) P e(n)+P e(k) P d(n) i ; \\
P e(n, k, 0) & =P e(n, k-2,0)+P e(n, k-3,0) \\
& =P d(k-2) P e(n)+P e(k-1) P d(n) i+P d(k-3) P e(n)+P e(k-2) P d(n) i \\
& =P d(k) P e(n)+P e(k+1) P d(n) i .
\end{aligned}
$$

With this, the veracity of Theorem 3.5 , is proven, through its application to $m=1,2,3, \ldots, k$, in the situation presented below:

$$
\begin{aligned}
P e(n, m, 0) & =P d(m) P e(n)+P e(m+1) P d(n) i \\
P e(n, m, 1) & =P d(m) P e(n)+P e(m+1) P d(n) i+2 P d(n) j \\
P e(n, m, 2) & =P d(m) P e(n)+P e(m+1) P d(n) i+3 P d(n) j \\
\vdots & \\
P e(n, m, k-3) & =P d(m) P e(n)+P e(m+1) P d(n) i+P e(k-2) P d(n) j \\
P e(n, m, k-2) & =P d(m) P e(n)+P e(m+1) P d(n) i+P e(k-1) P d(n) j ; \\
P e(n, m, k-1) & =P d(m) P e(n)+P e(m+1) P d(n) i+P e(k) P d(n) j ; \\
; & \\
P e(n, m, k) & =P e(n, m, k-2)+P e(n, m, k-3) \\
& +P d(m) P e(n)+P e(m+1) P d(n) i+P e(k-1) P d(n) j \\
& =P d(m) P e(n)+P e(m+1) P d(n) i+P e(k+1) P d(n) j .
\end{aligned}
$$

\section{The $n$-dimensional Perrin relations}

From the two-dimensional and three-dimensional relations discussed above, it is possible to generalise the insertion of imaginary units up to the $n$ order, thus obtaining the $n$-dimensional relations of the Perrin sequence. Thus, we have a generalised expression for the hypercomplex numbers described in the form $P e\left(n_{1}, n_{2}, \ldots, n_{n}\right)$, where $n$ represents the amount of imaginary variables inserted.

Theorem 4.1. Given $P e\left(n_{1}, n_{2}, \ldots, n_{n}\right)$ the numbers in Perrin $n$-dimensional form, with $n \in \mathbb{N}$ and the imaginary units represented by $\mu_{1}=i, \mu_{2}=j, \ldots, \mu_{n}$. Hence, they are given by:

$$
P e\left(n_{1}, n_{2}, \ldots, n_{n}\right)=\operatorname{Pe}\left(n_{1}\right) P d\left(n_{2}\right) \cdots P d\left(n_{n}\right)+P d\left(n_{1}\right) P e\left(n_{2}+1\right) \cdots P d\left(n_{n}\right) \mu_{1}+\cdots+P d\left(n_{1}\right) P d\left(n_{2}\right) \cdots P e\left(n_{n}+1\right) \mu_{n}
$$

Proof. Thus, it has already been demonstrated that the two-dimensional and three-dimensional relations are valid, which can be verified by the inductive process:

$$
\begin{aligned}
& P e\left(n_{1}, n_{2}\right)=\operatorname{Pe}\left(n_{1}\right) P d\left(n_{2}\right)+P e\left(n_{2}+1\right) P d\left(n_{1}\right) \mu_{1} \\
& \operatorname{Pe}\left(n_{1}, n_{2}, n_{3}\right)=\operatorname{Pe}\left(n_{1}\right) \operatorname{Pd}\left(n_{2}\right) \operatorname{Pd}\left(n_{3}\right)+\operatorname{Pd}\left(n_{1}\right) \operatorname{Pe}\left(n_{2}+1\right) \operatorname{Pd}\left(n_{3}\right) \mu_{1}+\operatorname{Pd}\left(n_{1}\right) P d\left(n_{2}\right) P e\left(n_{3}+1\right) \mu_{2} \\
& P e\left(n, n_{2}, n_{3}, n_{4}\right)=\operatorname{Pe}\left(n_{1}\right) P d\left(n_{2}\right) P d\left(n_{3}\right) P d\left(n_{4}\right)+P d\left(n_{1}\right) P e\left(n_{2}+1\right) P d\left(n_{3}\right) P d\left(n_{4}\right) \mu_{1} \\
& +\operatorname{Pd}\left(n_{1}\right) \operatorname{Pd}\left(n_{2}\right) \operatorname{Pe}\left(n_{3}+1\right) P d\left(n_{4}\right) \mu_{2}+\operatorname{Pd}\left(n_{1}\right) \operatorname{Pd}\left(n_{2}\right) \operatorname{Pd}\left(n_{3}\right) P e\left(n_{4}+1\right) \mu_{3} \\
& P e\left(n_{1}, n_{2}, n_{3}, \ldots, n_{n}\right)=\operatorname{Pe}\left(n_{1}\right) \operatorname{Pd}\left(n_{2}\right) \operatorname{Pd}\left(n_{3}\right) \cdots P d\left(n_{n}\right)+\operatorname{Pd}\left(n_{1}\right) \operatorname{Pe}\left(n_{2}+1\right) \operatorname{Pd}\left(n_{3}\right) \cdots P d\left(n_{n}\right) \mu_{1}+\cdots+ \\
& P d\left(n_{1}\right) P d\left(n_{2}\right) P d\left(n_{3}\right) \cdots P e\left(n_{n}+1\right) \mu_{n}
\end{aligned}
$$




\section{Conclusion}

Starting from the one-dimensional Perrin recursive model and the relationship between the Perrin numbers and the Padovan numbers, the recurrence two-dimensional, three-dimensional relations, and the inductive $n$-dimensional relation are explored concerning aspects of the complexity of the Perrin model. The process of complexity of the Perrin sequence was discussed through investigations around the addition of the imaginary unit, the dimensional increase, and its corresponding algebraic representations.

For future work, it is possible to develop mathematical properties around numbers $P e(n, m), P e(n, m, p)$ and $P e\left(n_{1}, n_{1}, n_{2}, \ldots, n_{n}\right)$, extend it to the integer indexes, and identify possible applications in the domain of science.

\section{Acknowledgements}

The authors would like to express their sincere thanks to the editor and the anonymous reviewers for their helpful comments and suggestions.

\section{Funding}

There is no funding for this work.

\section{Availability of data and materials}

Not applicable.

\section{Competing interests}

The authors declare that they have no competing interests.

\section{Author's contributions}

All authors contributed equally to the writing of this paper. All authors read and approved the final manuscript.

\section{References}

[1] M. C. dos S. Mangueira, R. P. M. Vieira, F. R. V. Alves, P. M. M. C. Catarino, A generalização da forma matricial da sequência de Perrin, ReviSeM, 5 (1) $(2020), 384-392$

[2] M. C. dos S. Mangueira, R. P. M. Vieira, F. R. V. Alves, P. M. M. C. Catarino, A generalized Perrin polynomial sequence and its two-dimensional recurrences, An. Stiint. Univ. Al. I. Cuza Iasi. Mat. (N.S.), in press.

[3] P. Seenukul, Matrices which have similar properties to Padovan $q$-matrix and its generalized relations, SNRU Journal of Science and Technology, 7 (2) (2015), 90-94.

[4] A. G. Shannon, P. G. Anderson, A. F. Horadam, Properties of Cordonnier, Perrin and van der Laan numbers, IJEMST, 37 (7) (2006), 825-831.

[5] K. Sokhuma, Matrices formula for padovan and perrin sequences, Appl. Math. Sci., 7 (142) (2013), 7093-7096.

[6] C. J. Harman, Complex Fibonacci numbers, The Fibonacci Quarterly, 19 (1) (1981), 82-86.

[7] R. R. de Oliveira, F. R. V. Alves, R. E. B. Paiva, Identidades bi e tridimensionais para os números de Fibonacci na forma complexa, C.Q.D.-Revista Eletrônica Paulista de Matemática, 11 (2) (2017), 91-106.

[8] R. R. de. Oliveira, Engenharia didática sobre o modelo de complexificação da sequência generalizada de Fibonacci: Relações recorrentes n-dimensionais e representações polinomiais e matriciais. Dissertação de Mestrado Acadêmico do Programa de Pós-graduação em Ensino de Ciências e Matemática do Instituto Federal de Educação, Ciência e Tecnologia do Ceará - IFCE - Campus Fortaleza, 2018.

[9] R. P. M. Vieira, F. R. V. Alves, P. M. M. C. Catarino, Relações bidimensionais e identidades da sequência de Leonardo, ReviSeM, 4 (2) (2019), 156-173. 